\title{
Modellings for Belief Change: Base Contraction, Multiple Contraction, and Epistemic Entrenchment (Preliminary Report)
}

\author{
Hans Rott \\ Fachgruppe Philosophie, Universität Konstanz, Postfach 5560, \\ D-7750 Konstanz, Germany
}

\begin{abstract}
This paper draws a distinction between the set of explicit beliefs of a reasoner, the "belief base", and the beliefs that are merely implicit. We study syntax-based belief changes that are governed exclusively by the structure of the belief base. In answering the question whether this kind of belief change can be reconstructed with the help of something like an epistemic entrenchment relation in the sense of Gärdenfors and Makinson [8], we extract several candidate relations from a belief base. The answer to our question is negative, but an approximate solution is possible, and in some cases the agreement is even perfect. Two interpretations of the basic idea of epistemic entrenchment are offered. It is argued that epistemic entrenchment properly understood involves multiple belief changes, i.e., changes by sets of sentences. Since none of our central definitions presupposes the presence of propositional connectives in the object language, the notion of epistemic entrenchment becomes applicable to the style of knowledge representation realized in inheritance networks and truth maintenance systems.
\end{abstract}

\section{Introduction}

\subsection{Representation of beliefs}

Our model of belief will be a simple one. A belief is represented by a sentence in some (regimented) language. Research done in Artificial Intelligence has recently lead to a revival of the logic of belief. It was felt that a clear distinction should be drawn between the explicit and the implicit beliefs of a reasoner $[14,18]$. The former ones are those that the reasoner would assent to if asked and for which he has some kind of independent warrant. The latter ones are those that follow, by some specified logic, from the set of explicit beliefs.

We distinguish a belief base, the set of explicit beliefs, from a belief set. A belief set is closed under logical consequences, it is a theory in the logician's sense. In general, we conceive of belief sets as generated by belief bases. Let us say that $H$ is a belief base for the belief set $K$ if and only if $K$ is the set of all logical consequences of $H$, i.e., if $K=C n(H)$.

We must make a decision what to count as a belief state. A belief state is that kind of thing, pre-theoretically understood, that is changed when we change our beliefs. As we cannot read off from a belief set $K$ which beliefs in it are the explicit ones, a belief state cannot be just a belief set. Should we then say that a belief state is modelled by a belief base $H$ ? Of course, we then have no problem in generating the full belief set, provided we have fixed an appropriate logic $C n$. So everything 
we could possibly want to know about the set of currently entertained beliefs can be answered if $H$ is known. However, as we shall see, there is a dynamical problem with this conception. In the sort of changes we shall consider, we cannot satisfy two desiderata at the same time: the desideratum that the changed belief state can be characterized by a belief base, and the desideratum that this belief base contains the set of explicit beliefs after the change has been effected. This is an unpleasant state of affairs which we shall have to put up with in this paper. Giving an answer to our question, we say that a belief state is a pair $\langle H, K\rangle$ such that $H$ is a belief base for $K$. However, the reader be warned that our change operations are not making belief states out of belief states in response to a certain input. We shall explain this in the next section.

Before doing that, let us delineate the object language and its logic. The logic of belief change, and especially the theory of epistemic entrenchment, has been discussed for a language with the expressiveness of propositional logic, including all its connectives $\neg, \wedge, \vee, \rightarrow$ and $\leftrightarrow$, as well as the truth and falsity constants $T$ and $\perp$. In contrast to this, we aim at reducing the linguistic prerequisites. Our considerations are to apply also to systems using severely restricted languages, as encountered e.g. in inheritance nets or truth (reason) maintenance systems.

Correspondingly, the logic governing our language has to obey only structural rules. We require that it is reflexive, monotonic, transitive, and compact. We refer to our logic either as a consequence operator $C n$ or as an inference relation $\vdash$, with the usual understanding that $\phi \in C n(H)$ iff $H \vdash \phi$. In the first notation our four requirements become

(R) $H \subseteq C n(H)$

(M) If $H \subseteq H^{\prime}$ then $C n(H) \subseteq C n\left(H^{\prime}\right)$

(T) $\quad C n(C n(H)) \subseteq C n(H)$

(C) If $\phi \in C n(H)$ then $\phi \in C n\left(H^{\prime}\right)$ for some finite subset $H^{\prime}$ of $H$

When we link our considerations to earlier work, we make use of connectives. Then the logic is further supposed to be supraclassical, i.e., what follows classically from a given premise set should follow from it in $C n$. We also assume that $C n$ satisfies the deduction theorem.

\subsection{Dynamics}

A belief change occurs if a belief state is changed in order to accommodate it to a certain input. In the case we are going to deal with, the input comes in the form of (explicit) beliefs. In the research program initiated by Alchourrón, Gärdenfors and Makinson ([3]; for excellent surveys, see [6] and [16]), belief states are identified with belief sets, and inputs are single sentences. Still working in broadly the same research program, Fuhrmann $[4,5]$ and Hansson $[9,10,11]$ offer modellings for two important generalizations. They investigate what happens when belief states are identified with belief bases (with belief sets as special cases) and when the input comes in sets of sentences (with singletons as special cases). In short, they generalize the theory of belief change to base changes and multiple changes. 
It is clear from the very beginning that the idea of base change is indeed compelling. True, it is reasonable to say that what an agent really believes is the belief set $K$, including the full set of his implicit beliefs. But it is at least as reasonable to think of belief change operations as acting on the set of explicit beliefs alone. After all, merely implicit beliefs have a secondary status, they are derived from the explicit ones. And if some of the explicit beliefs they depend on should have to give way, so should they! This is a foundationalist picture of belief revision and contrasts with the coherentist picture predominant in the current theory of belief revision $[7,13]$. We will endorse the philosophy of base change in this paper.

Again, it is a good idea to be ready for set-like inputs. But this issue does not seem to have the same philosophical force as base contraction. Philosophically, base change is an alternative to theory change, while multiple change is just an extension of singleton change. There seems to be no intimate connection between these two kinds of deviation from the original framework of Alchourrón, Gärdenfors and Makinson. However, we shall argue that multiple belief changes play a significant role in the analysis of base changes.

\subsection{Three types of belief change}

The simplest type of belief change is the addition of a new belief $\phi$ (or a set of beliefs) which is consistent with the old beliefs. In this case, we have no problem to identify the relevant operations. We can effect theory change through base change. Using the symbol ' + ', we define consistent additions as follows:

$$
\begin{gathered}
H+\phi=H \cup\{\phi\} \\
K+\phi=C n(K \cup\{\phi\})
\end{gathered}
$$

Notice that ' + ' has two different meanings here, depending on whether its first argument is supposed to be a belief base or a belief set. It is obvious how to generalize these definitions when the input comes in sets. However, the generalization will be far from obvious in the remaining cases, so we shall restrict ourselves to singleton inputs in the rest of this section.

The operation of accommodating a belief state to some input is considerably more difficult if the latter is inconsistent with the former. In this case, it is held that consistency should act as an integrity constraint for our belief system. For such belief-contravening additions, we shall adopt the following idea: In order to rationally include $\phi$ into the set $H$ (or $K$ ) of your beliefs, first make $H$ (or $K$ ) consistent with $\phi$, i.e., recant the commitment to $\neg \phi$, and then add $\phi$ consistently to the resulting set. It is common to use the term 'revision' to cover both consistent and belief-contravening additions, and to use the symbols ' $*$ ' for revisions and '-' for contractions. The above idea which is credited to Isaac Levi in the literature then becomes:

$$
\begin{gathered}
H * \phi=(H \dot{-} \neg \phi)+\phi=(H \dot{-} \neg \phi) \cup\{\phi\} \\
K * \phi=(K \dot{-} \neg \phi)+\phi=C n((K \dot{-} \neg \phi) \cup\{\phi\})
\end{gathered}
$$


This is the Levi identity, in its two versions for base and for theory change. One may think that the Levi identity is not of much help as long as we do not know how the contraction operation - behaves. This is right, but still it reduces the problem of finding suitable revision operations to the problem of finding suitable contraction operations. Philosophically, contraction appears to be the more fundamental operation. Like most authors in belief revision, we shall follow Levi's advice and study contractions in the following.

What is this fundamental interesting operation called 'contraction'? The contraction of a set of beliefs with respect to an input sentence $\phi$ is a subset of the original beliefs which does not logically imply $\phi$. (In a sense, "input sentences" for contractions are rather "output sentences".) The concept of logical consequence is obviously relevant here. In case we start with a belief set $K$, we should end up with another belief set $K \dot{-} \phi$ which is logically closed again. In contrast to the case of additions, we do not want to stipulate that the contracted belief set $K \dot{-} \phi$ can always be identified with the set of logical consequences of a new belief base $H \dot{-\phi}$. We will explain why presently.

\subsection{The basic idea of minimal change}

When forced to perform a belief change, it seems rational to preserve as many of the prior beliefs as possible. Many writers have embraced such a condition of minimal change (minimum mutilation, maximal conservativity, informational economy) for many different purposes [17]. We will use the label 'minimal change approach' as a proper name for that account of belief revision which covers at least maxichoice, full, and partial meet contraction in the sense of Alchourrón, Gärdenfors and Makinson [3].

This is the basic idea of minimal change: In order to contract a belief base $H$ (or a belief set $K$ ) with respect to $\phi$, look at the maximal subsets of $H$ (of $K$ ) which do not imply $\phi$. Since every piece of information is valuable, no gratuitous loss of beliefs is tolerated. Accordingly, we may say that a set $H_{1}$ of beliefs is better than (or preferred to) a set $H_{2}$ (relative to the belief base $H$ ) if $H_{1}$ preserves more explicit beliefs than $H_{2}$, that is, if $H_{2} \cap H$ is a proper subset of $H_{1} \cap H$. If $H_{1}$ and $H_{2}$ are subsets of $H$, this of course reduces to $H_{2} \subset H_{1}$. Generalizing a bit, we say that a set $\mathcal{H}_{1}$ of sets of sentences is better than (or preferred to) a set $\mathcal{H}_{2}$ of sets of sentences, in symbols $\mathcal{H}_{2} \sqsubset \mathcal{H}_{1}$, if for every $H_{2}$ in $\mathcal{H}_{2}$ there is an $H_{1}$ in $\mathcal{H}_{1}$ with $H_{2} \cap H \subset H_{1} \cap H$.

\section{Base contraction and multiple contraction}

In the following, the term 'base contraction' is not to be taken literally. What is changed is the theory $K=C n(H)$ generated by a base $H$. But how the theory is changed depends on the way it is axiomatized, on the form of $H$. For instance, while $H=\{p, q\}$ and $H^{\prime}=\{p \wedge q\}$ generate the same theory $K$, we expect that $K-p$ contains $q$ if $K$ is axiomatized by $H$, but that $q$ is lost if $K$ is axiomatized by $H^{\prime}$. In the latter case, $q$ is inseperable from $p$. Throughout this paper, we assume that syntactical information (the structure of explicit beliefs) is the sole mechanism controlling belief change. 
The minimal change approach is afficted with a decisive difficulty. In general, there is more than one solution to the minimal change problem, i.e., more than one maximal set of beliefs which does not imply $\phi$. Following Alchourrón and Makinson [1], we let $H \perp \phi$ denote the set of all maximal subsets of $H$ which fail to imply $\phi$. The point is that there is usually more than just one member in $H \perp \phi$. What then to do? Assuming that only the syntactical information provided by the base governs a theory's dynamical behaviour, we adopt an egalitarians's point of view. All elements of $H \perp \phi$ are to be treated equally. ${ }^{1}$

The bold or credulous option is maxichoice base change: In order to eliminate $\phi$ from $K$, choose one element of $H \perp \phi$ at random, and close under $C n$.

DEFINITION 1. Let $H$ be a base for $H$ and $\gamma$ be a (single-valued) choice function which selects, for every nonempty set $H \perp \phi$, an arbitrary element $H^{\prime}$ of $H \perp \phi$. Then the maxichoice base contraction over $K$ determined by $H$ and $\gamma$ is given by

$$
\psi \in K \dot{-} \phi \text { iff } \forall \phi \text { and } \gamma(H \perp \phi) \vdash \psi \text {, or } \vdash \phi \text { and } \psi \in K \text {. }
$$

Being maximally conservative, maxichoice contraction comes as close to the idea of minimal change as possible. However, if we do not have any information to govern the choice of some particular element of $H \perp \phi$, there is no guarantee that $\gamma$ selects "the right" one. Believers do not play dice. The arbitrariness of maxichoice contractions is avoided by the next model for belief revision.

The skeptical option is full meet base change: In order to eliminate $\phi$ from $K$, take all the elements of $H \perp \phi$, then close each under $C n$, and finally take the intersection. $^{2}$

Definition 2. For any base $H$ for $K$, the full meet base contraction over $K$ determined by $H$ is given by

$$
\psi \in K \dot{-} \phi \text { iff } \forall \phi \text { and } H^{\prime} \vdash \psi \text { for every } H^{\prime} \in H \perp \phi \text {, or } \vdash \phi \text { and } \psi \in K \text {. }
$$

Full meet contractions depart from the idea of minimal change, because the intersection of a set of maximal non-implying subsets is not itself a maximal nonimplying subset. However, the symmetrical consideration of each element of $H \perp \phi$ is required by our decision to let in no other information than is encoded in the structure of the explicit beliefs. Opting for full meet contraction thus means adhering to the equality of rights of the members in $H \perp \phi$.

Alchourrón and Makinson [1] have shown that both maxichoice and full meet contraction make good sense only if $H$ is a non-theory. So let us emphasize right at the beginning that it is indeed essential for the following constructions that we have at our disposal a differentiation between explicit and implicit beliefs. This is not only a distinction which is desirable intuitively, but also a technical prerequisite.

It would not be quite right to characterize our proposals as "theory change through base change" [5]. We do not want to stipulate that $K \dot{-} \phi=C n(H \dot{-} \phi)$

\footnotetext{
${ }^{1}$ This should not be confused with the idea that all elements of $H$ are equally well entrenched. In general they are not, according to Definition 10 below.

${ }^{2}$ Essentially the same method is applied by Veltman [24] and Kratzer [12] for the analysis of counterfactuals, and by Poole [20] for nonmonotonic reasoning.
} 
for some appropriate $H \dot{-\phi}$. Let us illustrate why. Consider $H=\{p, q\}$ and retract $p \wedge q$ from $K=C n(H) . H \perp(p \wedge q)=\{\{p\},\{q\}\}$, so under $K \dot{-} \phi=C n(H \dot{-} \phi)$, maxichoice would give us either $K \dot{-}(p \wedge q)=C n(\{p\})$ or $K \dot{-}(p \wedge q)=C n(\{q\})$, while full meet would give us $K \dot{-}(p \wedge q)=C n(\emptyset)$. Neither of these solutions seems satisfactory. Intuitively, $K \dot{-}(p \wedge q)=C n(\{p \vee q\})$ would be good. Assuming that one of $p$ and $q$ may be false, we should still cling to the belief that the other one is true. But $H^{\prime}=\{p \vee q\}$ is no base which can be constructed naturally from $\mathrm{H}$ it certainly does not record any explicit belief. So we give up the aim of getting $\langle K \dot{-} \phi, H \dot{-} \phi\rangle$ from $\langle K, H\rangle$ and stay content with the more modest aim of getting $K \dot{-\phi}$ from $K$ with the help of the belief base $H$. That is, $H$ is relevant, and indeed all that is relevant, for the construction of $K \dot{-} \phi$ from $K$, but $H$ will not get revised itself. Pictorially, instead of the desirable transition $\langle K, H\rangle \stackrel{\phi}{\longmapsto}\langle K \dot{-} \phi, H \dot{-\phi}\rangle$ we will study the transition $K \stackrel{\phi, H}{\longrightarrow} K \dot{-} \phi$. There will be no suggestion as to the contents of $H \dot{-} \phi$.

When inputs come in sets, we are presented with two different kinds of contraction. The aim of a pick contraction is to give up at least one element of a set S, while the aim of a bunch contraction is to give up every element of a set $\mathrm{S}$, both times with minimal mutilation of the original belief state. ${ }^{3}$ In conformity with the basic idea of minimal change, we again focus on maximal non-implying subsets of $H$.

Let $H \perp\langle S\rangle$ be the set of all maximal subsets of $H$ which do not imply every element of $S$, and $H \perp[S]$ the set of all maximal subsets of $H$ which do not imply any element of $S$. Clearly, $H \perp\{\{\phi\}\rangle=H \perp[\{\phi\}]=H \perp \phi$.

The concepts of maxichoice and full meet base contraction can be generalized naturally to cover pick and bunch contractions as well. As the case of maxichoice contractions is entirely analogous, we restrict ourselves to full meet contractions. Borrowing Fuhrmann's [4] symbols, we introduce

DEFinition 3. For any base $H$ for $K$, the pick and bunch versions of multiple full meet base contraction over $K$ are defined as follows:

$\dot{\psi} \in K-\langle S\rangle$ iff $H^{\prime} \vdash \dot{\psi}$ for every $H^{\prime} \in H \perp\langle S\rangle \neq \emptyset$, or $H \perp\langle S\rangle=\emptyset$ and $\psi \in K$.

$\psi \in K \dot{-}[S]$ iff $H^{\prime} \vdash \psi$ for every $H^{\prime} \in H \perp[S] \neq \emptyset$, or $H \perp[S]=\emptyset$ and $\psi \in K$.

From now on, we will drop curly brackets within pointed and square brackets, so $K \dot{-}[\{\phi, \psi\}]$ will simplify to $K \dot{-}[\phi, \psi]$, and $H \perp\{\{\phi, \psi\}\}$ to $H \perp\langle\phi, \psi\rangle$, etc.

\section{Epistemic entrenchment}

The concept of epistemic entrenchment has turned out to be a natural and fruitful instrument for the analysis of belief change $[6,7,8,15,21,22,23]$. 'Epistemic entrenchment' is just another word for comparative retractability. Intuitively, $\phi<\psi$ means that it is easier to discard $\phi$ than to discard $\psi$. We may call this the basic

\footnotetext{
${ }^{3}$ André Fuhrmann [4] was probably the first to study pick and bunch contractions. He called them choice and meet contractions. For danger of confusion with maxichoice and full meet contraction, we introduce new names.
} 
idea of epistemic entrenchment. Below we shall offer two interpretations of this idea in order to make it more precise.

Technically, epistemic entrenchment relations are known to have a number of characteristic properties. The basic postulates are

(EE1) $\quad T \nless T$

$\left(\right.$ EE2 $\left.^{\uparrow}\right) \quad$ if $\phi<\psi$ and $\psi \vdash \chi$, then $\phi<\chi$

$\left(\mathbf{E E 2}{ }^{\downarrow}\right) \quad$ if $\phi<\psi$ and $\chi \vdash \phi$, then $\chi<\psi$

$\left(\mathbf{E E 3}^{\uparrow}\right) \quad$ if $\phi<\psi$ and $\phi<\chi$, then $\phi<\psi \wedge \chi$

$\left(\mathbf{E E}^{\downarrow}{ }^{\downarrow}\right) \quad$ if $\phi \wedge \psi<\psi$, then $\phi<\psi$.
(Non-Triviality)

(Continuing Up)

(Continuing Down)

(Conjunction Up)

(Conjunction Down)

There is an equivalent and more economical set of postulates which does not mention any connective of the object language. First, we replace Non-Triviality by irreflexivity. Second, we note that postulates $\left(\mathrm{EE} 2^{\uparrow}\right)$ and $\left(\mathrm{EE} 3^{\uparrow}\right)$ taken together are equivalent to $\left(\mathrm{EE}^{\uparrow}\right)$, while $\left(\mathrm{EE} 2^{\downarrow}\right)$ and $\left(\mathrm{EE}^{\downarrow}{ }^{\downarrow}\right)$ taken together are equivalent to $\left(\mathrm{EE}^{\downarrow}\right)($ see $[23])$ :

$\left(\mathbf{E E}^{\uparrow}\right) \quad$ if $\phi<\psi$ for every $\psi$ in a non-empty set $S$ and $S \vdash \chi$, then $\phi<\chi$

$\left(\mathbf{E E}^{\downarrow}\right) \quad$ if $\phi<\psi$ and $\{\psi, \chi\} \vdash \phi$ then $\chi<\psi$.

The set of basic postulates may be supplemented by the following ones.

$$
\text { if } H \forall \perp \text {, then: } \perp<\phi \text { iff } H \vdash \phi
$$

(Minimality)

$$
\text { if } \forall \phi \text {, then } \phi<T
$$

(Maximality)

$$
\text { if } \phi<\psi \text {, then } \phi<\chi \text { or } \chi<\psi
$$

(Virtual Connectivity)

Again purely structural formulations of (EE4) and (EE5) are possible by substituting 'there is a $\psi$ such that $\psi<\phi$ (such that $\phi<\psi$ )' for ' $\perp<\phi^{\prime}$ ' (for ' $\phi<T$ '). For the motivation and discussion of all these postulates, see Gärdenfors and Makinson [8] and Rott [23]. Epistemic entrenchment relations are required to satisfy (EE1) $-\left(\mathrm{EE}^{\downarrow}{ }^{\downarrow}\right)$ in [23], and in addition (EE4) - (EE6) in [8]. (In fact, Gärdenfors and Makinson work with the non-strict relation $\leq$ which can be defined from the strict relation < by taking the converse complement.)

Given a relation of epistemic entrenchment, how can we get a contraction function from it? For the principal case, where $\phi \in K$ and $\phi<T$, the standard definition $[8,23]$ is

DEFINITION 4. For any relation < of epistemic entrenchment, the large EE-contraction with respect to $<$ is given by

$$
\psi \in K \dot{-} \phi \text { iff } \psi \in K \text {, and } \phi<\phi \vee \psi \text { or } \vdash \phi
$$

The presence of the disjunction $\phi \vee \psi$ here is somewhat mysterious (to say the least). An alternative idea was ventilated in Rott [21]: 
DEFINITION 5. For any relation < of epistemic entrenchment, the small EE-contraction with respect to $<$ is given by

$$
\psi \in K \dot{-\phi} \text { iff } \psi \in K \text {, and } \phi<\psi \text { or } \vdash \phi
$$

Both Definition 4 and Definition 5 make sure that $K \dot{-} \phi$ is a theory and that the contraction function - satisfies a number of rationality postulates. Large EEcontractions, but not small EE-contractions, satisfy the so-called postulate of recovery: $K \subseteq(K \circ \phi)+\phi$.

It follow's from (EE2 $2^{\uparrow}$ ) that $K \dot{-} \phi$ according to Definition 5 is a subset of $K \dot{-} \phi$ according to Definition 4--whence the names. Lindström and Rabinowicz [15, Section 5] argue convincingly that given an epistemic entrenchment relation $<$, any reasonable contraction of $K$ with respect to $\phi$ should result in a belief set which includes the small and is included in the large EE-contraction.

The basic idea of epistemic entrenchment is still very vague and ought to be made more precise. The first or competitive interpretation of it suggests to determine the relative ease of retracting a sentence by looking at the fate of $\phi$ and $\psi$ in a direct competition between $\phi$ and $\psi$. It reconstructs epistemic entrenchment from observed contraction behaviour $[8,23]$ :

DEFINITION 6. For any contraction function - over $K$, the epistemic entrenchment relation revealed by - is given by

$$
\phi<\psi \text { iff } \psi \in K-(\phi \wedge \psi) \text { and } \phi \notin K \dot{-}(\phi \wedge \psi) \text {. }
$$

Definition 6 yields extremely nice results for large EE-contraction functions over a theory $K$. If the contraction function - satisfies certain rationality postulates, then $<$ as obtained by Definition 6 is a relation of epistemic entrenchment from which we can recover - with the help of Definition 4 . And conversely, if $<$ is a relation of epistemic entrenchment, then - as obtained by Definition 4 satisfies certain rationality postulates and permits a reconstruction of $<$ with the help of Definition 6. Details can be found in Gärdenfors and Makinson [8] and Rott [23].

In [23], I emphatically adopt the idea that $K \dot{-}(\phi \wedge \psi)$ is to be interpreted as a multiple contraction, viz. the pick contraction with respect to $\{\phi, \psi\}$. Contracting $K$ with respect to $\phi \wedge \psi, I$ argued, is exactly the same as retracting at least one of $\phi$

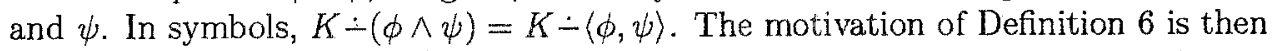
clear: if you have to give up either $\phi$ or $\psi$, and you give up $\phi$ and keep $\psi$, then $\psi$ has been more entrenched than $\phi$.

I do not see any intuitive reason for supposing that the identity $K \dot{-}(\phi \wedge \psi)=$ $K-\langle\phi, \psi\rangle$ is inadequate in some applications. Still it is good to be prepared for this possibility. Another motive for modifying Definition 6 is that we want to avoid explicit mentioning of particular connectives, in order to make the epistemic entrenchment approach applicable to restricted languages as encountered for instance in semantic networks. We take the motivation of Definition 6 seriously and suggest the following improvement:

DEFINITION 7. For any contraction function - over $K$, the epistemic entrenchment relation revealed by - is given by 


$$
\phi<\psi \text { iff } \psi \in K \dot{-}\langle\phi, \psi\rangle \text { and } \phi \notin K \dot{-}\langle\phi, \psi\rangle .
$$

This interpretation of the basic idea of epistemic entrenchment builds on the concept of pick contraction.

\section{Full meet base contraction as extended epistemic entrench- ment contraction}

In order to be able to deal with full meet base contractions in terms of epistemic entrenchment, we extend the basic idea of epistemic entrenchment to sets of sentences. From now on, ' $\langle S\rangle \ll\langle T\rangle$ ' is intended to mean that it is easier to discard some element of $S$ than to discard some element of $T$. ' $[S] \ll[T]$ ' is intended to mean that it is easier to discard all elements of $S$ than to discard all elements of $T$. We shall speak of extended epistemic entrenchment in the sequel, with the two types pick and bunch entrenchment.

Let us try to extend the competitive interpretation of epistemic entrenchment accordingly. For pick entrenchment, this is easy. The obvious suggestion is

$$
\langle S\rangle \ll\langle T\rangle \text { iff } T \subseteq K \dot{-}\langle S \cup T\rangle \text { and } S \nsubseteq K \dot{-}\langle S \cup T\rangle .
$$

But for bunch entrenchment, there is no sensible condition which can be formalized with the present means.

So we propose another understanding of--possibly extended - epistemic entrenchment. The second or minimal change interpretation of the basic idea of epistemic entrenchment builds on the basic idea of minimal change. It reads 'is easier' as 'does not require as great an informational loss as' or 'sacrifices fewer explicit beliefs than'. Formally, preference is identified with the proper subset relation ' $C$ '. Let us define the following version of extended epistemic entrenchment:

DEFINITION 8. For any base $H$ for $K$, the relation $\ll$ of bunch entrenchment generated by $H$ is defined by

$[S] \ll[T]$ iff for every $H^{\prime} \subseteq H$ such that $C n\left(H^{\prime}\right) \cap T=\emptyset$ there is an $H^{\prime \prime}$ such that $H^{\prime} \subset H^{\prime \prime} \subseteq H$ and $C n\left(H^{\prime \prime}\right) \cap S=\emptyset$, and $\forall \psi$ for every $\psi$ in $S$.

The following equivalent formulation is sometimes more convenient:

OBSERVATION 1 . Let $H$ be a base for $K$, and $\ll$ be the bunch entrenchment generated by $H$. Then $[S] \ll[T]$ iff for every $H^{\prime} \in H \perp[T]$ there is an $H^{\prime \prime} \in H \perp[S]$ such that $H^{\prime} \subset H^{\prime \prime}$, and $H \perp[S] \neq \emptyset$, i.e., iff $H \perp[T] \sqsubset H \perp[S]$, and $H \perp[S] \neq \emptyset$.

(The proofs of the observations are given in the full paper.) We spare the reader the analogous definition of pick entrenchment, and we do not want to enter into a discussion of the properties of $\ll$. We now observe that full meet base contractions allow an elegant characterization in terms of bunch entrenchment.

OBSERVATION 2. Let $H$ be a base for $K,-$ be the full meet base contraction determined by $H$, and $\ll$ be the relation of bunch entrenchment generated by $H$. Then $\psi \in K \dot{-} \phi$ iff $[\phi] \ll[\phi, \psi]$. 
We can directly represent full meet base contractions as extended EE-contractions in the following sense.

DEFINITION 9. For any relation « of bunch entrenchment, the EEE-contraction with respect to $\ll$ is given by

$$
\psi \in K-\phi \text { iff } \psi \in K \text {, and }[\phi] \ll[\phi, \psi] \text { or } \vdash \phi
$$

Like Definition 5, this definition is connective-free.

\section{Epistemic entrenchment generated by belief bases}

Given a base $H$ for $K$, we now try to find a more familiar, i.e., non-extended, relation of epistemic entrenchment without beforehand committing ourselves to a certain contraction method. We again exploit the basic idea of epistemic entrenchment. As it happens, the competitive and the minimal change interpretation of it can be unified in the present case. For the definition, we employ the latter one:

DEFINITION 10 . For any base $H$ for $K$, the relation of epistemic entrenchment generated by $H$ is given by

$$
\begin{gathered}
\phi<\psi \text { iff } \forall \phi \text { and for every } H^{\prime} \subseteq H \text { such that } H^{\prime} \forall \psi \text { there is an } H^{\prime \prime} \text { such that } \\
H^{\prime} \subset H^{\prime \prime} \subseteq H \text { and } H^{\prime \prime} \forall \phi .
\end{gathered}
$$

This is a singleton version of Definition 8. Clearly, it is a negative interpretation of epistemic entrenchment, focussing on the ways to discard a belief. It is intuitively well-motivated. Roughly, $\psi$ is more entrenched than $\phi$ iff for every way of discarding $\psi$ there is a better way of discarding $\phi$. As a special case of Observation 1 , we take down

OBservation 3. Let $H$ be a base for $K$, and $<$ be generated by $H$. Then $\phi<\psi$ iff $\forall \phi$ and for every $H^{\prime} \in H \perp \psi$ there is an $H^{\prime \prime} \in H \perp \phi$ such that $H^{\prime} \subset H^{\prime \prime}$, i.e., iff $\forall \phi$ and $H \perp \psi \sqsubset H \perp \phi$.

So $\psi$ is more entrenched than $\phi$ if for every "best" way of discarding $\psi$ there is a still better "best" way of discarding $\phi$. More exactly, in terms of maxichoice contraction functions, if for every $\gamma$ there is a $\gamma^{\prime}$ such that the maxichoice base contraction of $K$ with respect to $\phi$ determined by $\gamma^{\prime}$ properly includes the maxichoice base contraction of $K$ with respect to $\psi$ determined by $\gamma$.

In the following, we trace some of the implications of this definition. First, we verify that Definition 10 generates a relation of epistemic entrenchment in the generalized sense of Rott [23], but not in the standard sense of Gärdenfors and Makinson [8]. Then we show that for full meet base contractions the two interpretations of the basic idea of epistemic entrenchment coincide. In the next section, we show that full meet base contractions, which are EEE-contractions characterized by Definitions 8 and 9 , can be interpolated by means of small and large EE-contractions based on the epistemic entrenchment relation generated by the base. Although the approximation cannot in general be strengthened to an identity, sometimes a perfect agreement can be attained.

It is easy to verify that the relation < defined in Definition 10 has the following properties: 
OBSERVATION 4. For every belief base $H$, the relation $<$ generated by $H$ satisfies (EE1) - (EE3〉) and (EE4) - (EE5), but it does not satisfy (EE6).

That is, Definition 10 does not yield an epistemic entrenchment relation in the sense of Gärdenfors and Makinson [8], but it does yield an epistemic entrenchment relation in the less demanding sense of Rott [23].

If pick contractions are formalized as in Definition 3, Definition 10 turns out to be equivalent with the first interpretation of the basic idea of epistemic entrenchment as formalized in Definition 7. In the present context, the competitive and the minimal change interpretations of epistemic entrenchment coincide.

Observation 5 (CoINCIDENCE Lemma). Let $H$ be a base for $K$, let $<$ be the epistemic entrenchment relation generated by $H$ and $<^{\prime}$ be the epistemic entrenchment relation revealed by the pick version of multiple full meet base contraction. Then $\phi<\psi$ iff $\phi<^{\prime} \psi$.

\section{Full meet base contractions as approximated by epistemic entrenchment contractions}

Suppose that the epistemic entrenchment relation < is generated by the belief base $H$ for $K$. We wonder about the relation between full meet base contractions generated by $H$ (or alternatively, by Definitions 8 and 9 ) on the one hand and the large and small EE-contractions based on < on the other. Is it possible to get what we got by extended epistemic entrenchment above with the help of singleton epistemic entrenchment? As for large EE-contractions, the answer must be negative, because they are known to satisfy the recovery postulate, which base contractions notoriously do not.

It turns out that singleton epistemic entrenchment is insufficient in general, but an approximation by upper and lower bounds is possible. The entrenchment relation determined by a belief base $H$ with the help of Definition 10 allows us to follow the above-mentioned recommendation of Lindström and Rabinowicz. We can interpolate full meet base changes according to Definition 2, i.e., EEE-changes according to Definitions 8 and 9 , by large and small EE-changes according to Definitions 4 and 5 .

OBSERVATION 6 (INTERPOLATION LEMMA). Let $H$ be a base for $K$ and $<$ be the entrenchment relation generated by $H$. Furthermore, let - be the full meet base contraction function determined by $H$, let $\dot{-}_{1}$ be the small and $\dot{-}_{2}$ be the large $E E-$ contraction with respect to $<$. Then

$$
K \dot{-}_{1} \phi \subseteq K \dot{-} \phi \subseteq K \dot{-}_{2} \phi .
$$

The converse inclusions are not valid.

In a couple of cases, the correspondence between full meet base contraction and epistemic entrenchment contraction is perfect, if the latter is to mean large EEcontraction based on the relation $<$ generated by the belief base $H$. There are at least three ways of equivalence. We list them in increasing importance. 
Theories. The first case is when the base $H$ is already a belief set, i.e., when $H=K$. However, this case is of limited relevance. The epistemic entrenchment relation generated by $K$ is nearly empty if $K$ is a theory, because then $K^{\prime} \in K \perp \psi$ and $K^{\prime \prime} \in K \perp \phi$ imply $K^{\prime} \not \subset K^{\prime \prime}$, unless $K^{\prime \prime}$ equals $K$. So in this case, $\phi<\psi$ according to Definition 10 can hold only if either $\psi \in K$ and $\phi \notin K$, or $\vdash \psi$ and $\forall \phi$. This corresponds to a well-known trivialization result of Alchourrón and Makinson [1, Observation 2.1] for full meet contractions of theories.

Nebel's blown-up contractions. The second case in point is when full meet base contraction is supplemented with a mechanism to enforce the recovery postulate. This is basically the suggestion of Nebel [19]:

\section{Definition 11. For any base $H$ for $K$, the blown-up contraction - is given by}

$$
\begin{gathered}
\psi \in K \dot{-} \text { iff }\left(K \dot{-1}_{1} \phi\right) \cup\{\phi \rightarrow \chi: \chi \in H\} \vdash \psi \text {, where } K \dot{-1}_{1} \phi \text { is the full meet base } \\
\text { contraction determined by } H .
\end{gathered}
$$

The set $\operatorname{Rec}=\{\phi \rightarrow \chi: \chi \in H\}$ is a recovery ticket which allows one to "undo" a base contraction with respect to $\phi$. It is easy to check that on Definition $11, K=$ $(K \dot{-} \phi)+\phi$, for every $\phi$ in $K$. But since clearly $\neg \phi$ implies every element of Rec, and Rec in turn implies $\phi \rightarrow \psi$ for every $\psi$ in $K$, we find that $\psi \in K \dot{-} \phi$ according to Definition 11 iff $\phi \vee \psi \in K-\phi$ according to Definition 2. It is not difficult to see that this is equivalent to saying the $\phi$ is in the large EE-contraction of $K$ based on the epistemic entrenchment relation generated by $H$.

Revisions based on the Levi identity. Thirdly, the correspondence is perfect if a contraction is only an intermediate for a revision constructed with the help of the Levi identity. Since, by Levi and the deduction theorem, $\psi$ is in $K * \phi$ iff $\phi \rightarrow \psi$ is in $K-\neg \phi$, we have to check $K\llcorner\neg \phi$ only for sentences of the form $\phi \rightarrow \psi$. But clearly, for every EE-relation $<, \neg \phi<(\phi \rightarrow \psi)$ is equivalent to $\neg \phi<\neg \phi \vee(\phi \rightarrow \psi)$, so Definitions 4 and 5 are equivalent for sentences of the form $\phi \rightarrow \psi$ in $K-\neg \phi$. Hence, by the Interpolation Lemma, either form of EE-revision is identical with full meet base revision.

\section{Conclusion}

The aim of this report has been to provide an illustration for the versatility of the concept of epistemic entrenchment, to apply epistemic entrenchment to belief states ("bases") which are not supposed to be logically closed, and to further the intuitive understanding of epistemic entrenchment and its relation to multiple contraction.

Our starting point has been a fixed belief base $H$ generating a belief set $K$. Our concern is "syntax-based" belief change, or belief change determined by belief bases, and we assume that the structure of $H$ is the sole information governing the changes of $K$. We have given a reformulation of full meet base contractions as extended EE-contractions: Definition 2 is equivalent to the combination of Definitions 8 and 9 . This representation depends on an extension of epistemic entrenchment to sets of sentences ("bunch entrenchment"). We elaborated on the basic idea of epistemic entrenchment as comparative retractability by giving it two different readings. The usual "competitive" interpretation was distinguished from what we called the "minimal change interpretation" of the phrase " $\psi$ is harder to discard than $\phi$ '. 
We proposed a method of extracting an epistemic entrenchment relation $<$ from a belief base $H$. Discovering that Definition 10 is equivalent to the combination of Definitions 3 and 7 , we observed a confluence of the two interpretations of epistemic entrenchment (the "Coincidence Lemma"). It was demonstrated that upper and lower bounds of full meet base contractions can be specified in the form of large and small EE-contractions based on the relation < generated by the belief base (the "Interpolation Lemma").

Since the publication of Gärdenfors's Knowledge in Flux, relations of epistemic entrenchment have been known to be interdefinable with belief contractions. For theory change by singletons, the following transitions are standard in the literature:

$$
\begin{aligned}
& \phi<\psi \text { iff } \psi \in K \dot{-}(\phi \wedge \psi) \text { and } \phi \notin K \dot{-}(\phi \wedge \psi) \\
& \psi \in K \dot{-} \phi \text { iff } \psi \in K, \text { and } \phi<\phi \vee \psi \text { or } \vdash \phi
\end{aligned}
$$

To my mind, there is no denying that these bridge principles are the pivotal points of an illuminating and well-developed theory of belief change $[6,8,21,22,23]$. However, the occurrences of ' $\wedge$ ' and ' $V$ ' are somewhat mysterious. This is why I suggest a more transparent way to think of the interdefinability between epistemic entrenchment and belief change.

$$
\begin{aligned}
& \phi<\psi \text { iff } \psi \in K \dot{-}\langle\phi, \psi\rangle \text { and } \phi \notin K \dot{-\langle\phi, \psi\rangle} \\
& \text { (Definition 7) }
\end{aligned}
$$

Full meet base specialization: if $\dot{-}$ is the full meet base contraction determined by $H$, then, by Observations 3 and $5, \phi<\psi$ is definable by $H \perp \psi \sqsubset H \perp \phi$

Singleton reformulation: $\psi \in K \dot{-}(\phi \wedge \psi)$ and $\phi \notin K \dot{-}(\phi \wedge \psi)$

$$
\psi \in K \dot{-} \phi \text { iff } \psi \in K \text {, and }[\phi] \ll[\phi, \psi] \text { or } \vdash \phi
$$

(Definition 9)

Full meet base specialization: if $\ll$ is the bunch entrenchment generated by $H$, then, by Observation 2 , full meet base contraction coincides with EEE-contraction, and $\psi \in K \dot{-} \phi$ is definable by $H \perp[\phi, \psi] \sqsubset H \perp[\phi]$

Singleton interpolation: in so far as $\phi<\psi$ implies $[\phi] \ll[\phi, \psi]$, and this in turn implies $\phi<\phi \vee \psi$, large and small EE-contractions can serve as upper and lower bounds of EEE-contractions

Our deviation from the standard account is clear. We invoke sets with two elements as arguments for contraction operations and entrenchment relations. More specifically, we replace, in the direction from belief change to epistemic entrenchment, the contractions with respect to conjunctions by pick contractions, and in the direction from epistemic entrenchment to belief change, the entrenchments of disjunctions by bunch entrenchments.

What is the reward for this exercise? First and foremost, we get a better understanding of the relevant interrelations. They sometimes happen to reduce to the standard definitions. But what is really meant by the latter is, I submit, precisely what is made explicit by the new definitions. In one direction, I should think there is virtually no difference: $K \dot{-}(\phi \wedge \psi)$ seems to be intuitively identifiable with $K \dot{\sqcup}\langle\phi, \psi\rangle$. 
In the other direction, however, it is only the restricted context of theory change by singletons that makes our new definition reduce to the old one: $[\phi] \ll[\phi, \psi]$ may-and must! - then be identified with $\phi<\phi \vee \psi$.

Secondly, we manage without reference to any particular connective of the object language. Thus the theory of epistemic entrenchment becomes applicable to systems using a severely restricted language. For instance, we can speak of the entrenchment of the nodes in inheritance nets or reason maintenance systems (also called "truth maintenance systems"). There ought to be a corresponding connective-free formulation of the so-called Gärdenfors postulates for contraction operations. The obvious suggestion is to replace occurrences of ' $K-(\phi \wedge \psi)$ ' by ' $K-\langle\phi, \psi\rangle$ '. The elimination of connectives, however, works only for belief contractions. Belief revisions constructed according to the Levi identity make use of negations, and there does not seem to be a straightforward way to avoid this. ${ }^{4}$

At last, we should like to give two warnings. The connective-free formulation of epistemic entrenchment relations and theory contractions is only a by-product of this paper, slightly improving on the presentation in [23]. It is not necessary for the analysis of syntax-based belief change which turns essentially on the syntactical structure of the items in a belief base. There is no immediate transfer of insights from belief base update to updates in inheritance networks or reason maintenance systems ("RMSs") with their unstructured "nodes". It may be expedient for some purposes to identify RMS "justifications" with Horn clauses. But this certainly does not suffice for nonmonotonic systems. Our $C n$ is supposed to be monotonic.

Multiple contraction and extended epistemic entrenchment have been found to be an appropriate means for analyzing base contraction. However - this is the second warning-, the conepts of multiple contraction and extended epistemic entrenchment themselves, cut loose from the special context of maxichoice and full meet base contraction, are still very much in need of a thoroughgoing analysis. This is evidently beyond the scope of the present paper.

\section{References}

[1] Alchourrón, Carlos, and David Makinson (1982): "On the logic of theory change: Contraction functions and their associated revision functions", Theoria 48, 14-37.

[2] Alchourrón, Carlos, and David Makinson (1985): "On the logic of theory change: Safe contraction", Studia Logica 44, 405-422.

[3] Alchourrón, Carlos, Peter Gärdenfors and David Makinson (1985): "On the logic of theory change: Partial meet contraction and revision functions", Joumal of Symbolic Logic 50, 510-530.

[4] Fuhrmann, André (1988): Relevant Logics, Modal Logics, and Theory Change, $\mathrm{PhD}$ thesis, Australian National University, Canberra.

[5] Fuhrmann, André (1991): "Theory contraction through base contraction", Journal of Philosophical Logic 20, 175-203.

[6] Gärdenfors, Peter (1988): Knowledge in Flux: Modeling the Dynamics of Epistemic States, Bradford Books, MIT Press, Carnbridge, Mass.

${ }^{4}$ Thanks to an anonymous referee for pointing this out. 
[7] Gärdenfors, Peter (1990): "The dynamics of belief systems: Foundations vs. coherence theories", Revue Internationale de Philosophie 44, 24-46.

[8] Gärdenfors, Peter, and David Makinson (1988): "Revisions of knowledge systems using epistemic entrenchment", in Proceedings of the Second Conference on Theoretical Aspects of Reasoning about Knowledge, Moshe Vardi ed., Los Altos, CA: Morgan Kaufmann, pp. 83-95.

[9] Hansson, Sven Ove (1989): "New operators for theory change", Theoria 55, 114-132.

[10] Hansson, Sven Ove (1991): Belief base dynamics, Doctoral Dissertation, Uppsala University.

[11] Hansson, Sven Ove (1992): "Reversing the Levi identity", Journal of Philosophical Logic, forthcoming.

[12] Kratzer, Angelika (1981): "Partition and revision: The semantics of counterfactuals", Journal of Philosophical Logic 10, 201-216.

[13] Harman, Gilbert (1986): Change in View, Bradford Books, MIT Press, Cambrige, Mass.

[14] Levesque, Hector J. (1984): "A logic of implicit and explicit belief", Proceedings 3rd National Conference on Artificial Intelligence, Austin TX, pp. 198-202.

[15] Lindström, Sten, and Wlodzimierz Rabinowicz (1991): "Epistemic entrenchment with incomparabilities and relational belief revision", in André Fuhrmann and Michael Morreau eds., The Logic of Theory Change, Springer-Verlag, Lecture Notes in Artificial Intelligence 465, Berlin, pp. 93-126.

[16] Makinson, David (1985): "How to give it up: A survey of some formal aspects of the logic of theory change", Synthese 62, 347-363.

[17] Makinson, David (1992): "The five faces of minimal change", unpublished manuscript.

[18] McArthur, Gregory L. (1988): "Reasoning about knowledge and belief: A survey", Computational Intelligence 4, 223-243.

[19] Nebel, Bernhard (1989): "A knowledge level analysis of belief revision", in Ronald Brachman, Hector Levesque and Raymond Reiter eds., Principles of Knowledge Representation and Reasoning. Proceedings of the 1st International Conference, Morgan Kaufmann, San Mateo, Ca., pp. 301-311.

[20] Poole, David (1988): "A logical framework for default reasoning", Artificial Intelligence 36, 27-47.

[21] Rott, Hans (1991): "Two methods of constructing contractions and revisions of knowledge systems", Journal of Philosophical Logic 20, 149-173.

[22] Rott, Hans (1992a): "On the logic of theory change: More maps between different kinds of contraction function", in Peter Gärdenfors ed., Belief Revision, Cambridge University Press, Cambridge, pp. 122-141.

[23] Rott, Hans (1992b): "Preferential belief change using generalized epistemic entrenchment", Journal of Logic, Language and Information 1, 45-78.

[24] Veltman, F. (1976): "Prejudices, presuppositions and the theory of counterfactuals", in Jeroen Groenendijk and Martin Stokhof eds., Amsterdam Papers of Formal Grammar, Vol. I, Centrale Interfaculteit, Universiteit Amsterdam, pp. 248-281. 